each catalyst independently controls the formation of one stereocentre, and as such all possible diastereomers of the product are accessible simply by choosing the right combination of catalysts. Their initial report focused on the reaction of branched aldehydes (activated with a chiral amine) with allyl alcohols (activated by a chiral iridium complex). Now, the same team of researchers have expanded the concept to the more challenging linear aldehyde substrates.

These new starting materials present two major challenges. Unlike the earlier, more hindered, substrates linear aldehydes are prone to self-condensation under the reaction conditions. Perhaps more importantly, the reaction products now incorporate an enolizable stereocentre such that, even if the product is formed selectively, epimerization under the reaction conditions would be feasible. A chiral secondary amine was selected to activate the aldehyde - based on precedent from related reactions - and initial experiments were focused on the selection of an acidic promoter for the activation of the allyl alcohol component. Careful tuning of this acidity was necessary because weak acids allowed undesired sidereactions to be competitive and stronger acids had a detrimental effect on selectivity. Ultimately, dimethyl hydrogen phosphate was found to provide the optimal acidity. Furthermore, the anticipated epimerization of the product in the presence of this acid was found to be slow relative to the rate of product formation.

The selective formation of all four possible diastereomers was demonstrated and the scope of the reaction investigated. Several different aryl-allyl carbinols were tested but, as yet, suitable conditions have not been identified for the reaction of aliphatic allyl alcohols. An ester and a phthalimide group were tolerated in the aldehyde component. Finally the practicality of the method was demonstrated in a concise synthesis of (-)-paroxetine - an antidepressant.

POLYMERSOMES

\section{A light alteration}

Angew. Chem. Int. Ed. http://dx.doi.org/10.1002/ anie.201310589 (2014)

The desire to mimic biological systems such as cells has resulted in interest in self-assembled block copolymer vesicles, or 'polymersomes', for drug delivery or as artificial organelles. Key to their utility is the ability to encapsulate and transport molecules, either in the central water pool,

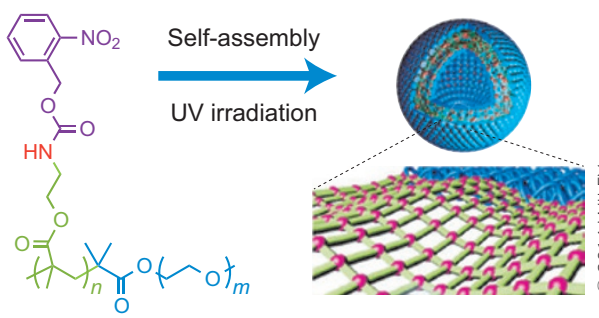

or sequestered in their hydrophobic bilayer membrane. Unlike lipid vesicles found in nature, however, the bilayer membranes of such polymersomes are often relatively impermeable, hindering the release of encapsulated molecules. Many strategies to overcome this impermeability have been developed, but they often require multiple synthetic steps or result in the destabilization of the polymersome.

Now, Shiyong Liu and colleagues from the University of Science and Technology of China in Hefei have developed a strategy that can simultaneously enhance the permeability of a polymersome membrane, whilst also crosslinking it to provide further stability. They do this by using a lightinduced reaction that leads to a hydrophilic network of channels that permeates the membrane. Inducing a hydrophobic-tohydrophilic transition in the membraneforming block of the copolymer renders the polymer completely hydrophilic, and should result in disassembly of the polymersome. However, Liu and co-workers used photolabile carbamates to effect the hydrophobic-to-hydrophilic transition. On irradiation with UV light the carbamates degrade to expose amine groups that react immediately with nearby esters in the membrane and effectively 'stitch' it together before the polymersome can disassemble - creating the hydrophilic channels in the process (pictured).

Release of both a hydrophobic dye from the membrane and a hydrophilic drug from the water pool was shown to be promoted by UV irradiation, with only minimal release through the non-irradiated membranes. Large dextran and enzyme molecules remained trapped within the polymersome after irradiation, showing that release is size-dependent. The intriguing possibility of photoregulated enzyme activation was also demonstrated, with an encapsulated enzyme functioning only after UV irradiation - not due to any change in the enzyme itself, but rather the ability of the substrate and product molecules to penetrate the polymersome membrane.

Written by Stuart Cantrill, Stephen Davey and Claire Hansell.

\section{blog $_{\text {roll }}$ 贯}

\section{The human element}

\section{Chemists are people too.}

What is behind that amazing paper you just read? A great idea, hard work and, possibly, good writing skills. But that's only a small part of the story. That paper is soaked (hopefully metaphorically) with the blood, sweat and tears of the postdocs and students who have worked tirelessly on the project. Beneath that aseptically written scientific paper there is an army of young researchers for many of whom their daily job is an emotional rollercoaster. From the excitement of a good result, to the umpteenth failed experiment and the depression of a rejection.

Many researchers can relate to the feelings of doubt, anxiety and stress shared by Beth Haas who blogs at Casual Science. Beth's words "I am good at what I do, and I am so very lucky to do something I love" (http://go.nature.com/QfiD5L) should be engraved in every fumehood, to be gazed upon when dark periods descend. Dr. K. of Trockeneisbombe offers her point of view (http://go.nature.com/ FYkUra) on work-life balance, which is that putting your life on hold is definitely not a good idea; friends and hobbies are important. And the one-year-old blog post (http://go.nature.com/8wqyNI) from See Arr Oh at Just Like Cooking about the mental toll of grad school should be required reading for all researchers working in academia.

And finally, the fight against chemophobia continues in the blogosphere. 'A picture is worth a thousand words' is perhaps nowadays better expressed as 'infographics are worth a thousand words'. James Kennedy's poster on chemicals in an all-natural banana has gone viral (http://go.nature. $\mathrm{com}$ (OJEvk8), reaching the mainstream media. Other eye-catching infographics can be seen on the blog Compound Interest (http://go.nature.com/ GkRMwR), ranging from the chemistry of smartphones to chocolate.

Written by Vittorio Saggiomo, who blogs at http://www.labsolutely.org/ 